\title{
Rayonnement laser : risques oculaires et normes de protection
}

\author{
D. COURANT*, C. CHAPEL*, S. PAOLACCI-RIERA**, J.-C. PÉROT**
}

(Manuscrit reçu le 24 aoùt)

RÉSUMÉ Les propriétés optiques des tissus biologiques conditionnent de manière importante le risque d'atteinte par le faisceau laser. Les rayonnements, émis dans l'ultraviolet mais aussi l'infrarouge $B$ et $C$, représentent un danger pour le segment antérieur de l'oil. Selon la longueur d'onde, le rayonnement est absorbé préférentiellement ou exclusivement par la cornée ou le cristallin. Les seuils de lésion les plus faibles concernent les rayonnements compris entre 400 et $1400 \mathrm{~nm}$, susceptibles de traverser les milieux oculaires et d'être focalisés sur la rétine. Les valeurs limites d'exposition ou EMP, recommandées par les normes de sécurité laser, sont établies en fonction de la longueur d'onde, de la durée de l'exposition, de la fréquence de répétition des impulsions et de la dimension de la source. La rapide évolution technologique des lasers entraîne une révision constante des normes de protection. Le prochain amendement à la norme européenne EN 60825-1/A2 comportera des limites d'exposition aux impulsions inférieures à la nanoseconde, une modification des limites d'exposition aux sources laser continues ainsi qu'une nouvelle classification des lasers susceptible de s'harmoniser avec les futures normes américaines ANSI et FDA/CDRH.

ABSTRACT Laser radiation: ocular risks and safety standards.

The risk for biological tissues to be damaged by a laser beam is mainly determined by their optical properties. Laser radiations emitted in ultraviolet and infrared $B$ and $C$ represent a hazard for the anterior segment of the eye. Depending the wavelength, laser radiation is absorbed by the cornea, the lens or both. The lower damage thresholds are reported at the retinal level, in the spectral range of 400 to $1400 \mathrm{~nm}$. The exposure limit values, recommended by laser safety standards, are evaluated with wavelength, exposure duration, pulse repetition frequency and the size of the visual angle subtending the source. The fast evolution of laser products induces a continuous revision of guidelines. Next revision of European standard EN 60825-1/A2 will include subnanosecond limit values, changes in the continuous wave exposure limits and a new classification of laser products susceptible to be in agreement with next ANSI and FDA/CDRH laser safety standards.

* Commissariat à l'énergie atomique, DSV/DRR/LRP, BP 6, 92265 Fontenay-aux-Roses, France.

** Direction des centres d'expertise et d'essai, CTA/LOT, 16 bis avenue Prieur de la Côte d'Or, 94114 Arcueil, France. 


\section{Introduction}

Le laser a des applications particulièrement variées grâce à ses caractéristiques exceptionnelles : monochromaticité, faible divergence, cohérence spatiale et temporelle, très grande énergie ou très grande puissance, rayonnement continu ou par impulsions ultracourtes et longueur d'onde accordable. Ses paramètres induisent sur les matériaux et les tissus biologiques des effets qui ne sont pas sans risque. C'est pourquoi des limites d'exposition ont été déterminées et la fabrication ou l'utilisation des appareils réglementée par un ensemble de normes. Les dangers présentés par le faisceau laser sont liés aux atteintes oculaires et cutanées mais nous n'évoquerons que le risque oculaire qui est uniquement concerné par la prochaine révision des normes de sécurité.

\section{Les risques oculaires}

L'œil étant l'organe de la vision, le récepteur privilégié des rayonnements optiques visibles, il est normal qu'il soit l'organe le plus sensible à l'effet des rayonnements laser. Les propriétés optiques des milieux oculaires vont conditionner de manière importante le risque d'atteinte par le faisceau.

\subsection{Dans l'ultraviolet (180 à $400 \mathrm{~nm}$ )}

De 180 à $300 \mathrm{~nm}$, des surexpositions au faisceau peuvent induire des irritations de la conjonctive et de la cornée qui peuvent se révéler très douloureuses (Sliney, 1986).

De 300 à $400 \mathrm{~nm}$, une cataracte ou perte de la transparence du cristallin peut s'associer aux dommages de la cornée. Au-delà de $330 \mathrm{~nm}$, les cataractes observées semblent, pour la plupart, induites par des expositions répétées ou prolongées (Sliney et Wolbarsht, 1980; Taylor et al., 1988).

\subsection{Dans le visible (400 à $760 \mathrm{~nm}$ ) et l'infrarouge A (760 à $1400 \mathrm{~nm})$}

Dans la gamme de longueur d'onde de 400 à $1400 \mathrm{~nm}$, le plus grand danger est le dommage rétinien. Ces rayonnements sont susceptibles de traverser les milieux oculaires et d'être focalisés sur la rétine. L'augmentation de l'éclairement énergétique de la cornée à la rétine suit approximativement le rapport de la surface pupillaire à celle de l'image rétinienne. En ambiance obscure, le diamètre maximum de la pupille peut atteindre $7 \mathrm{~mm}$. Cette valeur est considérée, dans les normes, comme l'ouverture ou le diaphragme limite représentant la pire condition de vision. La dimension d'une image rétinienne, correspondant à une telle pupille, est de 10 à $20 \mu \mathrm{m}$. En considérant la diffusion des milieux intraoculaires et les aberrations de la cornée, 
l'augmentation de l'éclairement énergétique entre la cornée et la rétine est de l'ordre de $2 \times 10^{5}$. C'est ce gain optique considérable qui permet à des faisceaux de puissance relativement faible d'endommager la rétine. Une lésion rétinienne peut être induite par une impulsion de quelques microJoules ou par le faisceau d'un simple laser $\mathrm{HeNe}$, à émission continue, de quelques milliWatts seulement.

Si un faisceau laser intense est focalisé sur la rétine, seule une petite fraction de l'énergie est absorbée par les pigments des cônes et des bâtonnets. La majorité du rayonnement est absorbée par la mélanine contenue dans l'épithélium pigmentaire rétinien. Dans la région maculaire, une partie de l'énergie correspondant aux longueurs d'onde de 400 à $500 \mathrm{~nm}$ est également absorbée par le pigment maculaire. L'énergie absorbée produit un échauffement local et une brûlure de l'épithélium pigmentaire mais aussi des photorécepteurs adjacents. Cette lésion peut entraîner une perte de la vision. Des lésions photochimiques peuvent également être induites au niveau de l'épithélium pigmentaire (Ham et al., 1979 ; Noell, 1980). Les seuils de brûlure rétinienne sont en général beaucoup plus bas pour les émissions de faible longueur d'onde : il existe au moins un facteur 10 entre l'énergie nécessaire pour provoquer une lésion avec un laser à argon $(488 \mathrm{~nm})$ ou avec un laser au néodyme (1064 nm) (Ham et al., 1976). La brûlure de l'épithélium pigmentaire, qui résulte de l'absorption du rayonnement, endommage les photorécepteurs situés au voisinage immédiat et peut, selon l'importance de l'exposition, s'étendre aux autres couches de la rétine (Marshall, 1970 ; Guéneau et al.,1986). A l'examen du fond d'œil, la gravité de la lésion varie de la dépigmentation, à peine perceptible, à l'hémorragie envahissant le vitré (Borland et al., 1978 ; Court et al., 1984 ; Courant et al. 1986, 1989a, 1994). L'épithélium pigmentaire peut se reformer par division des cellules demeurées intactes alors que les cellules nerveuses ne se divisent pas (Marshall et Mellerio, 1967). Les cônes et les bâtonnets dont les corps cellulaires sont lésés dégénèrent et meurent. À cet endroit, la fonction visuelle est définitivement perdue. Ceci montre l'importance de la localisation de l'image.

L'acuité visuelle et la vision des couleurs sont l'apanage de la macula, petite zone de 2 à 2,5 mm de diamètre, creusée en son centre d'une fossette d'environ $0,15 \mathrm{~mm}$ de diamètre : la fovéa. C'est à ce niveau que se fait l'image du point de fixation et il est aisé de concevoir que sa destruction, même partielle, soit particulièrement invalidante. Une atteinte couvrant $2^{\circ}$ du champ visuel et centrée sur la fovéa, suffit à réduire l'acuité visuelle de $50 \%$; si la lésion couvre $5^{\circ}$, l'acuité sera diminuée d'environ $70 \%$. Cette diminution de l'acuité visuelle est toujours le signe d'une atteinte de la région maculaire, qu'elle soit la conséquence d'une lésion anatomique ou d'un simple éblouissement (Harwerth et Sperling, 1971 ; Sperling, 1980; Zwick et al., 1983; Zwick, 1989). À l'inverse, une lésion de la rétine périphérique, qui ne provoque qu'un éblouissement passager, ne modifie pas l'acuité ou le sens chromatique. De même, une lésion de la rétine périphérique induite par un rayonnement du proche infrarouge, ne produisant pas d'éblouissement, peut passer inaperçue et n'être révélée qu'à l'occasion d'un examen du fond d'oil ou du champ visuel (Lund, 1998). 
La cornée, le cristallin, l'humeur aqueuse et le vitré sont bien sûr transparents aux rayonnements visibles et, dans une moindre mesure, aux rayonnements invisibles du proche infrarouge, toutefois, la faible absorption du cristallin entre 900 et $1400 \mathrm{~nm}$ suffit à induire une cataracte.

\subsection{Dans l'infrarouge B et C $\left(1400\right.$ à $\left.10^{6} \mathrm{~nm}\right)$}

De 1400 à $3000 \mathrm{~nm}$, les dommages induits au niveau oculaire sont des brûlures de la cornée mais aussi des pertes de transparence du cristallin, qui seraient secondaires à l'absorption du rayonnement infrarouge par l'iris (Sliney et Wolbarsht, 1980 ; Wolbarsht, 1991). De 3000 à $10^{6} \mathrm{~nm}$, les lésions oculaires sont essentiellement des brûlures de l'épithélium antérieur de la cornée.

\section{Les normes de protection}

Les normes européennes, donc françaises, de sécurité laser EN 60825 sont basées sur les publications de la Commission électrotechnique internationale (CEI) et les amendements du Comité européen de normalisation électrotechnique (CENELEC). La norme fondamentale EN 60825-1 (IEC, 1993) définit des valeurs limites d'exposition ou expositions maximales permises (EMP) au rayonnement laser. Les EMP sont issues des recommandations de l'International Commission on Non Ionizing Radiation Protection (ICNIRP, 1996), souvent prises comme références par de nombreux organismes pour l'établissement des limites d'exposition aux rayonnements non ionisants.

\subsection{Les valeurs limites d'exposition}

Dans l'état actuel de nos connaissances, les valeurs limites d'exposition sont censées représenter le niveau de rayonnement laser auquel les personnes peuvent être exposées sans risque de dommage immédiat ou à long terme. Les limites d'exposition sont évaluées, au niveau de la cornée, en fonction de la longueur d'onde, de la durée de l'exposition et de la fréquence de répétition des impulsions. Les limites d'exposition les plus faibles correspondent au risque rétinien, défini pour la plage spectrale de 400 à $1400 \mathrm{~nm}$. Dans cette gamme de longueur d'onde, l'influence de la dimension de l'image sur le seuil de lésion introduit des limites différentes pour les sources ponctuelles et étendues (Beatrice et Frisch, 1973; Courant et al., 1989b; Zuclich et al., 1999).

La très grande majorité des expositions dangereuses au rayonnement laser concerne la vision directe du faisceau correspondant à la vision d'une source ponctuelle. Cependant, des lasers de forte puissance peuvent engendrer, par réflexion sur 
des cibles diffusantes ou des matériaux translucides, des conditions dangereuses de vision de sources étendues. Les limites d'exposition pour la vision de telles sources s'appliquent aux longueurs d'onde susceptibles d'atteindre la rétine lorsque l'angle apparent $\alpha$ sous lequel est vue la source depuis l'œil de l'observateur, est supérieur à un angle $\alpha_{\min }$. Ces limites sont déterminées en multipliant les limites d'exposition aux sources ponctuelles par un facteur de correction proportionnel à la dimension de la source.

Dans le cas de vision d'une source ponctuelle, comme celle engendrée par un faisceau bien collimaté, le danger est virtuellement indépendant de la distance entre la source et l'œil car l'image rétinienne est supposée être une tache, de diffraction limitée, d'environ 10 à $20 \mu \mathrm{m}$ de diamètre. L'angle apparent minimum $\left(\alpha_{\min }\right)$ correspond à une tache sur la rétine de $25 \mu \mathrm{m}$ de diamètre environ.

Dans le cas de vision d'une source étendue, le danger varie en fonction de la distance entre l'œil et la source car la diffusion thermique de l'énergie, à l'intérieur des images rétiniennes, devient moins efficace à mesure que leur dimension s'accroît (Sliney et Wolbarsht, 1980; Sliney, 1996; Courant et al., 1989b; Cain et al., 1998). Cette influence du diamètre de l'image rétinienne sur le seuil de lésion n'existe pas dans le cas des lésions photochimiques induites par les rayonnements de 400 à $600 \mathrm{~nm}$.

\subsection{La révision des expositions maximales permises (EMP)}

Les récentes propositions de l'ICNIRP (2000) concernant les limites d'exposition au rayonnement laser sont incluses dans le projet de révision $\mathrm{A}_{2}$ de la norme 60825-1. Ces révisions concernent plusieurs paramètres dans la plage de 400 à $1400 \mathrm{~nm}$.

\subsubsection{Les expositions subnanosecondes $\left(t<10^{-9} s\right)$}

À l'origine, il n'existait pas de limite d'exposition pour les durées inférieures à la nanoseconde $\left(10^{-9} \mathrm{~s}\right)$ en raison des controverses sur les mécanismes impliqués et la grande disparité des rares résultats expérimentaux (Goldman et al., 1975, 1977 ; Taboada et Gibbons, 1978; Bruckner et Taboada, 1982; Birngruber et al., 1989). Ces derniers ne permettaient pas de prédire l'évolution des seuils de lésion en fonction de la durée de l'impulsion. La seule recommandation, effectuée de manière très préliminaire, fut de limiter l'éclairement énergétique des expositions subnanosecondes à celui établi pour une durée de 1 nanoseconde (IEC, 1993). Des études des lésions rétiniennes induites par des impulsions provenant de laser fonctionnant en mode déclenché dans le domaine de la picosecond $\left(10^{-12} \mathrm{~s}\right)$ et de la femtoseconde $\left(10^{-15} \mathrm{~s}\right)$ ont été réalisées dans plusieurs programmes de recherche. Le développement des limites a été difficile en raison de l'implication potentielle de différents mécanismes d'interaction du rayonnement laser avec les tissus biologiques (Cain et al., 1996, 1997; Roach et al., 1994; Jacques et al., 1996; Thompson 


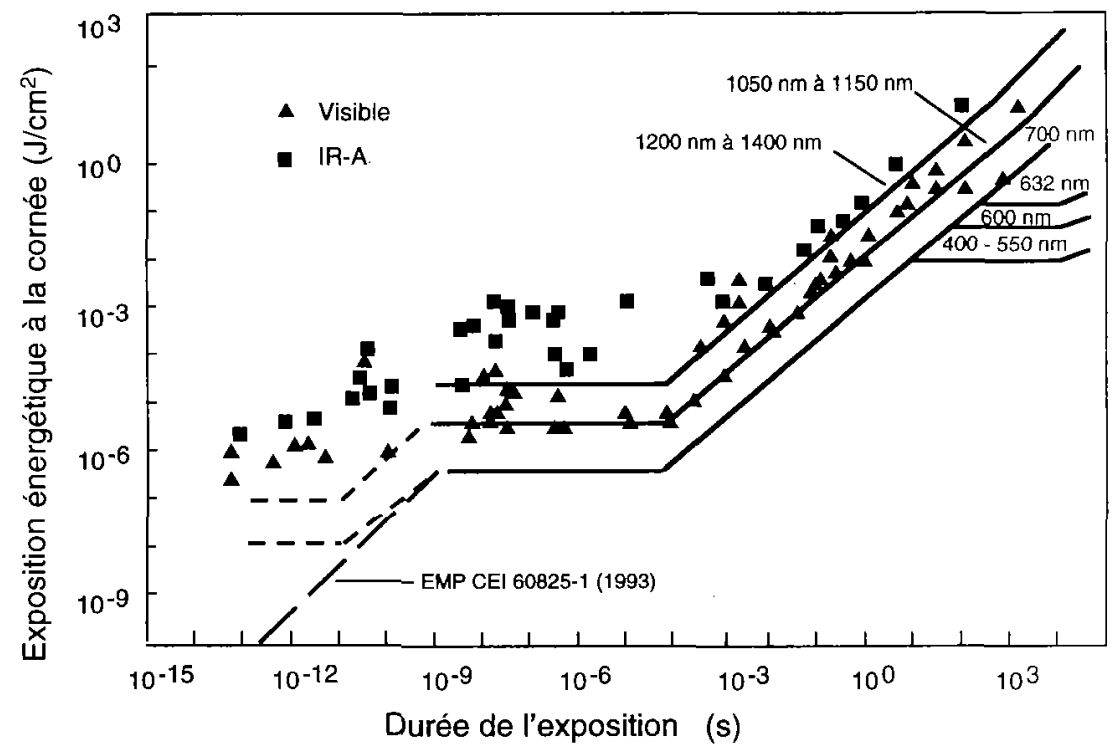

Fig. 1. - Seuils de dommage à la rétine $\left(D E_{50 \%}\right)$ et limites d'exposition oculaire entre 400 et $1400 \mathrm{~nm}$ (IEC 60825-1, 1993 ; ICNIRP, 1996). Les traits discontinus représentent les limites d'exposition proposées pour les durées inférieures à $10^{-9} \mathrm{~s}$.

Retinal injury thresholds and ocular exposure limits between 400 and $1400 \mathrm{~nm}$ (IEC 60825-I, 1993; ICNIRP, 1996). Exposure limites proposed for subnanosecond exposures are represented with dashed lines.

et al., 1996 ; Toth et al., 1997). Les premières données expérimentales montraient que les mécanismes de dommage non linéaires ne sont pas influencés par la longueur d'onde, la durée de l'impulsion et la dimension de l'image rétinienne de la même manière que les mécanismes de dommage thermique ou thermo-acoustique. Des études histologiques de grandes et petites plages de rétine, exposées à des impulsions variant de quelques picosecondes à quelques centaines de femtosecondes et à différentes longueurs d'onde, ont montré toutefois que les effets observés sur la rétine sensorielle ont toujours pour origine les granules de mélanine de l'épithélium pigmentaire rétinien (Toth et al., 1996, 1997; Courant et al., 1998, 1999a,b). D'autres études ont permis de tester différents modèles de mécanisme lésionnel basés sur des mécanismes de cavitation et de variation de pression (Cain et al., 1997, 1998; Gertsman et al., 1996; Hammer et al., 1997 ; Strauss et al., 1997 ; Rockwell et al., 1997 ; Lin et Kelly, 1998 ; Brinkmann et al., 1999). La base de données scientifiques est devenue maintenant suffisante pour recommander, pour la première fois, des limites d'exposition à des durées d'impulsions aussi courtes que 100 femtosecondes (Roach et al., 1999). La figure 1 montre les niveaux de seuil de dommage 
provenant de plusieurs études et la dérivation des nouvelles limites. La diminution des limites d'exposition dans le domaine picoseconde est basée sur des calculs théoriques liés à l'effet d'auto-focalisation par les milieux oculaires; le meilleur ajustement est réalisé par une fonction de type $\mathrm{f}\left(\mathrm{t}^{0,75}\right.$ ) (Cain et al., 1997; Roach et al., 1999). Dans le domaine femtoseconde, d'autres mécanismes d'optique non linéaire, qui nécessitent des études plus approfondies, semblent contribuer au processus lésionnel (Rockwell et al., 1997).

L'ICNIRP prévoit d'établir des limites d'exposition pour la peau et la cornée lorsque des données seront disponibles dans les régions spectrales concernées. Il en est de même pour les expositions aux durées d'impulsions comprises entre 10 et $100 \mathrm{fs}$, dans les régions du spectre visible et du proche infrarouge.

\subsubsection{Expositions à des impulsions répétitives}

Il existe peu de données concernant les effets des impulsions multiples et les normes recommandent de prendre des précautions dans l'évaluation de l'exposition au rayonnement (Griess et al., 1980). Les propositions de l'ICNIRP conservent les règles précédemment utilisées. Toutefois, le facteur de correction d'additivité thermique, qui est fonction du nombre d'impulsions délivrées, ne s'applique qu'aux limites concernant les lésions thermiques. Les impulsions délivrées en un temps inférieur à $t_{\min }$ sont assimilées à une impulsion unique ; la valeur de $t_{\min }$ varie avec la longueur d'onde. Il n'est pas sûr que cette formulation qui satisfait un mécanisme essentiellement thermique soit applicable à des trains d'impulsions très courtes susceptibles de mettre en jeu d'autres mécanismes (Courant et al., 1999 a,b).

\subsubsection{Expositions continues}

Les tentatives pour appliquer les limites d'exposition établies pour la vision des lasers continus aux diodes émettant de la lumière (DEL) ou aux diodes lasers ont révélé certaines incohérences de ces limites (Sliney, 1997). En conséquence, le groupe de travail mandaté par l'ICNIRP a également été chargé d'étudier la validité des limites actuelles aux lasers continus. Les défauts observés sont liés à l'élaboration des premiers EMP aux lasers continus, censés couvrir à la fois les lésions rétiniennes induites par des mécanismes thermiques et photochimiques à l'aide d'une formulation unique. Comme les dommages induits d'une manière thermique ou photochimique varient différemment en fonction du temps, de la longueur d'onde et de la dimension de l'image rétinienne, d'immenses facteurs de sécurité avaient été introduits dans certaines conditions (Sliney, 1999). De plus, le facteur de correction pour la vision des sources étendues créait des EMP encore plus conservatifs pour les expositions continues du fait qu'il soit uniquement dérivé des effets thermiques. L'influence des mouvements oculaires n'était pas pris en compte dans ces deux mécanismes de dommage - en particulier dans le cas des très longues durées de vision (Ness et al., 2000). 


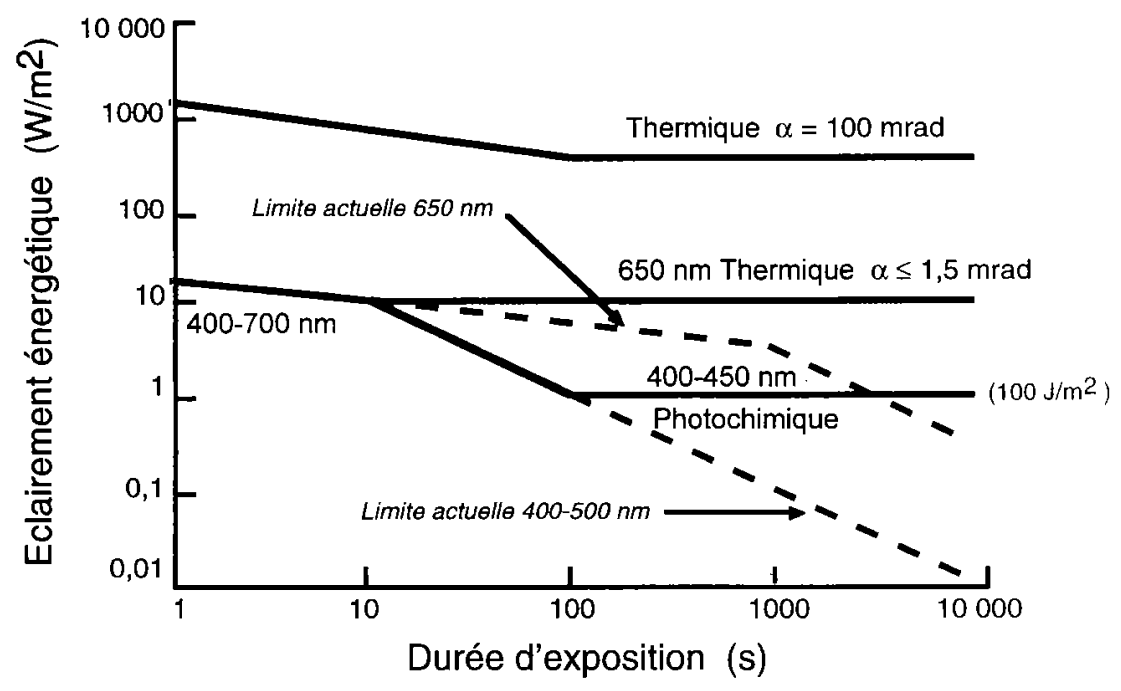

Fig. 2. - Comparaison entre les limites d'exposition actuelles et les propositions de l'ICNIRP et de la CEI (IEC 60825-I/A2) pour la vision des lasers à émission continue. La valeur limite recommandée pour 400 à $450 \mathrm{~nm}$, entre 10 et $100 \mathrm{~s}$, est indépendante de la dimension de la source en raison d'un mécanisme lésionnel photochimique, contrairement à celle donnée pour $650 \mathrm{~nm}$, qui est influencée par un mécanisme thermique.

Comparison between current exposure limits and proposal from ICNIRP and IEC (60825I/A2) for continuous wieving lasers. The damaging photochemical mechanism explains that the limit value recommended for 400 to $450 \mathrm{~nm}$, from 10 to $100 \mathrm{~s}$, is independent of sourcesize at the opposite of the limit given for $650 \mathrm{~nm}$, where thermal mechanism plays a role only.

La proposition de l'ICNIRP Ophthalmic Biophysics Task Group est de créer des EMP comprenant deux limites : l'une pour la photorétinite, l'autre pour la lésion thermique (ICNIRP, 2000) (Fig. 2). Pour se protéger contre le risque photochimique, les nouvelles EMP établies pour les longueurs d'onde de 400 à $600 \mathrm{~nm}$ se réfèrent à la valeur limite de $100 \mathrm{~J} \mathrm{~m}^{-2}$, actuellement utilisée pour les «dangers de la lumière bleue » incohérente, pour les longueurs d'onde de 400 à $450 \mathrm{~nm}$ et à cette même valeur, affectée d'une fonction d'ajustement exponentielle, entre 450 et $600 \mathrm{~nm}$ (ICNIRP, 1997). Pour la protection contre le risque thermique, les EMP s'appliquent à la totalité de la région de danger rétinien, de 400 à $1400 \mathrm{~nm}$. Les EMP ont été révisées pour les durées d'exposition supérieures à $10 \mathrm{~s}$ dans le cas de vision de petites images $\left(\alpha \leq \alpha_{\min }\right)$ et pour les expositions supérieures à $100 \mathrm{~s}$ dans le cas des sources étendues $(\alpha>100 \mathrm{mrad})$. Il n'y a pas de changements pour les expositions comprises entre 1 ns et $10 \mathrm{~s}$ dans les conditions de vision des sources de dimensions minimum, en revanche, les limites pour la vision des sources de grandes dimensions sont modifiées aux durées supérieures à $0,3 \mathrm{~s}$. 


\subsection{La classification des lasers}

La norme française NF EN 60825-1 comprend des prescriptions de fabrication, des règles d'utilisation et une classification des appareils à laser. En effet, les fabricants de lasers sont tenus de classer leurs appareils destinés à la vente en fonction du risque présenté par le faisceau en utilisation normale. Le rayonnement sortant effectivement de l'appareil est comparé à des limites d'émission accessibles, fondées sur les limites d'exposition, qui délimitent les classes. L'intérêt de ces classes est pour l'utilisateur de préciser immédiatement, au seul vu de la classe, les dangers d'un système laser sans avoir à recourir à des mesures radiométriques et des comparaisons avec les valeurs limites d'exposition. Le fabricant d'appareils à laser est tenu selon la classification d'incorporer certains dispositifs de sécurité et de respecter un certain nombre de spécifications techniques.

Les appareils à laser sont groupés en 4 classes générales; les limites d'émissions accessibles (LEA) sont spécifiées pour chacune d'elles.

- La classe 1 : Ce sont les lasers intrinsèquement sans danger. Les LEA ne peuvent en aucun cas dépasser les limites d'exposition les plus restrictives.

- La classe 2: Ce sont des dispositifs à faible puissance émettant un rayonnement visible $(400$ à $700 \mathrm{~nm}$ ). Ils ne sont pas intrinsèquement sans danger, mais la protection de l'œil est normalement assurée par le réflexe palpébral. La puissance ou l'énergie émise par ces systèmes est limitée aux LEA de la classe 1 pour des durées d'exposition allant jusqu'à 0,25 s. Pour un laser continu, la LEA est de $1 \mathrm{~mW}$.

- La classe 3: Elle comprend des lasers de puissance moyenne pour lesquels le risque incendie est négligeable et pour lesquels l'exposition momentanée de la peau n'entraîne pas de dommage. Elle est divisée en deux sous-classes:

- la classe 3A: Les réflexions diffuses ne sont pas dangereuses et la vision directe du faisceau n'est dangereuse que si elle est supérieure à $0,25 \mathrm{~s}$ ou si elle se fait à travers des instruments d'optique. La LEA est de $5 \mathrm{~mW}$ en continu ou de 5 fois la LEA de classe 2 pour les lasers impulsionnels dans le spectre visible. Pour les autres régions spectrales, le rayonnement ne doit pas dépasser 5 fois les LEA de la classe 1.

- la classe 3B : La vision directe dans le faisceau est toujours dangereuse et la vision des sources étendues est considérée sans risque si le temps d'exposition est limité à $10 \mathrm{~s}$ et la distance d'observation est supérieure à $13 \mathrm{~cm}$. Les lasers continus ne peuvent dépasser $500 \mathrm{~mW}$ et l'exposition énergétique provenant des lasers impulsionnels doit être inférieure à $10^{5} \mathrm{~J} \mathrm{~m}^{-2}$.

- La classe 4 : Elle concerne les lasers de puissance suffisante pour créer des dommages cutanés et oculaires, en vision directe ou diffuse et qui peuvent aussi constituer un danger d'incendie. Ce sont des lasers toujours dangereux dont l'utilisation exige de très grandes précautions.

La classification est assurée par le fabricant qui en est responsable. La classe doit être indiquée dans le guide d'utilisation et sur l'appareil lui-même grâce à un étiquetage réglementé. 
Ce premier schéma de classification est utilisé par les normes CEI et CENELEC mais aussi par les normes US (ANSI, 1993 ; FDA/CDRH, 1985) et les prescriptions imposées aux fabricants sont identiques. Toutefois, une différence existe entre les normes US et CEI dans l'estimation du risque correspondant à chaque classe selon que les conditions de vision considèrent l'hypothèse d'un risque zéro ou d'un risque raisonnablement prévisible. Ces deux concepts sont à l'origine de quelques petites différences dans les conditions de mesure des LEA. Ainsi, dans le système US, la mesure des LEA avec une grande ouverture ne s'effectue que s'il existe une chance raisonnable d'utiliser des optiques. Dans la CEI et l'approche européenne, le programme d'essai requis suppose que la vision à l'aide d'optiques est toujours concevable. En conséquence, le même laser peut être classé $3 \mathrm{~A}$ par la norme ANSI Z136.1 et 3B par la norme CEI 60825-1.

\subsection{Le nouveau schéma de classification}

La prochaine révision de la norme CEI devrait mettre un terme à la controverse qui existe depuis les années 1990. L'amendement $A_{2}$ à la publication 60825-1 comportera de nouvelles limites d'exposition mais aussi un nouveau schéma de classification qui devrait satisfaire les partisans du «risque zéro» et du «raisonnablement prévisible» (Tab. I). Ce nouveau schéma sera également incorporé dans la prochaine mise à jour de la norme ANSI Z136.1.

\section{TABLEAU I}

Nouveau schéma de classification des lasers des classes 1, 2 et 3 .

Modified classification scheme for lasers of classes 1, 2 and 3.

1. Les lasers de très faible risque, qui seraient sans danger dans les conditions d'utilisation raisonnablement prévisibles, seront en classes 1 et $1 \mathrm{M}$ :

- classe I : «à sécurité oculaire» même en cas de vision à l'aide d'optique,

- classe 1M : «à sécurité oculaire» sauf en cas de vision à l'aide d'optique (jusqu'à la LEA de la classe $3 \mathrm{~B}$ ).

2. Les lasers, présentant un faible risque en raison d'une réaction d'aversion, seront en classes 2 et $2 \mathrm{M}$ :

- classe 2 : «à sécurité oculaire » en condition de vision momentanée (comme l'actuelle classe 2), - classe $2 \mathrm{M}$ : «à sécurité oculaire » en condition de vision momentanée sans l'aide d'optique (jusqu'à la LEA de la classe 3B).

3. Les lasers correspondants à la zone de transition seront en classe 3 :

- en classe 3R « exigences réduites », comme ceux qui satisfont actuellement la définition U.S. de la classe $3 \mathrm{~A}$ (lasers visibles de 1 à $5 \mathrm{~mW}$ ). Ceci s'appliquerait à toutes les longueurs d'onde supérieures à $302 \mathrm{~nm}$,

- en classe 3B tous les lasers correspondant à I'ancienne classe 3B excepté ceux correspondant à la classe $3 R$. 
La répercussion de la nouvelle classification sur les lasers existants sera minime car très peu d'appareils correspondent aux nouvelles classes. Tous les appareils à laser de l'actuelle classe 1 de la CEI resteront en classe 1 . Il en sera de même pour la majorité des lasers de classe 1 US, seuls quelques rares cas devraient correspondre à la nouvelle classe $1 \mathrm{M}$. Tous les appareils actuels de classe 2 resteront en classe 2 ou $2 \mathrm{M}$ si le faisceau est fortement divergent comme celui d'une diode laser. Tous les appareils actuels de la classe 3A US, classés 3B par la CEI comme les systèmes d'alignement à laser ou les pointeurs, seront renommés classe $3 \mathrm{R}$. Les appareils à laser de classe 3 A US et CEI avec un faisceau dilaté, qui sont rares en dehors de l'utilisation par les militaires ou à l'extérieur, seront maintenant en classes $1 \mathrm{M}$ et $2 \mathrm{M}$. Les fabricants n'auront aucune modification des spécifications requises pour leurs produits.

\section{Conclusion}

La sécurité du rayonnement laser est un aspect récent de la radioprotection qui sera encore soumis à de nombreuses modifications du fait de l'évolution technologique des lasers. Des limites d'exposition seront modifiées, d'autres comme pour les impulsions subnanosecondes, seront créées en fonction de nouvelles utilisations ou de nouveaux paramètres du faisceau. La validité, sinon l'efficacité, des futures limites d'exposition dépendra, pour une grande part, de la connaissance exacte des effets des rayonnements laser sur l'organisme et de leurs conséquences. C'est dans cet esprit que la révision des limites d'exposition au rayonnement laser s'est montrée nécessaire après un examen plus approfondi des conditions de vision des lasers continus. Cette révision a permis d'améliorer la validité des limites mais aussi d'appliquer les normes de sécurité laser aux sources incohérentes et aux diodes. Le nouvel amendement à la norme CEI devrait contribuer à rendre plus logique la classification des lasers en fonction du risque et permettre l'harmonisation des normes de sécurité laser au début de ce nouveau millénaire.

\section{RÉFÉRENCES}

ANS1 (1993) American national standard for the safe use of lasers, Standard Z136.1. American National Standard Institute Inc., New York.

Beatrice E.S., Frisch G.D. (1973) Retinal laser damage threshold as a function of image diameter, Arch. Environ. Health 27, 322-326.

Birngrüber R., Puliafito C.A., Gawande A., Lin W.-Z., Schoenlein R.W., Fujimoto J.G. (1989) Femtosecond laser-tissue interactions: retinal injury studies, Invest. Ophthalmol. 46, 1836-1844.

Borland B.G., Brennan D.H., Marshall J., Viveash J.P. (1978) The role of fluorescein angiography in the detection of laser-induced damage to the retina: A threshold study for Q-switched, neodymium and ruby lasers, Exp. Eye Res. 27, 471- 493. 
Brinkmann R., Rögener J., Lin C.P., Roider J., Birngruber R., Hüttmann G. (1999) Selective RPE-photodestruction: mechanism of cell damage by pulsed laser irradiance in the ns to $\mu$ s time regime In: Laser-tissue interaction X, January 22-24, San Jose, SPIE Vol. 3601 (Jacques S.L., Katzir A., Eds), pp. 59-65. Bellingham.

Brückner R., Taboada J. ( 1982) Retinal tissue damage induced by 6 picosecond $530 \mathrm{~nm}$ laser light pulses, Appl. Optics 21, 365-367.

Cain C.P., DiCarlo C.D., Rockwell B.A., Kennedy P.K., Noojin G.D., Stolarski D.J., Hammer D.X., Toth C.A., Roach W.P. (1996) Retinal damage and laser-induced breakdown produced by ultrashort-pulse lasers, Graefe's Arch. Clin. Exp. Ophthalmol. 234, S28-S37.

Cain C.P., Noojin G.D., Hammer D.X., Thomas R.J., Rockwell B.A. (1997) Artificial eye for in vitro experiments of laser light interaction with aqueous media, J. Biomed. Optics 2, 88-94.

Cain C.P., Toth C.A., Noojin G.D., Stolarski D.J., Payne D.P., Rockwell B.A. (1998) Visible lesion threshold dependancy on retinal spot size for ultrashort laser pulses in the near infrared. In: Lasertissue interaction IX, January 26-28, San Jose, SPIE Vol. 3254 (Jacques S.L., Katzir A, Eds), pp. 126-129. Bellingham.

Courant D., Court L., Abadie B., Brouillet B., Garcia J., Paradis J.-L., Perot J.-C., (1986) Experimental determination of a laser retinal lesion threshold produced by a single pulse in the visible spectrum. In: lst Symp. on Laser Biological Effects and Exposure Limits, November 24-28, Paris, (Court L., Duchêne A., Courant D., Eds), pp. 157-174, CEA/BDP, Fontenay-aux-Roses.

Courant D., Court L., Abadie B., Brouillet B. (1989a) Retinal damage thresholds from single pulse laser exposures in the visible spectrum, Health Phys. 56, 637-642.

Courant D., Court L., Sliney D.H. (1989b) Spot-size dependence of laser retinal dosimetry. In: Dosimetry of Laser Radiation in Medicine and Biology (Muiller G.J., Sliney D.H., Eds), pp. 156-165. SPIE Press, Washington.

Courant D (1994) Ocular and cutaneous risks after laser exposure. In: Limites d'exposition aux rayonnements non ionisants, May 25-26 Paris, (Court L., Lambroso J., Eds), pp. 137-152. SFRP, Paris.

Courant D., Fritsch P., Naudy-Vivès C., Le Naour H., Pérot J.-C., Garcia J., Dormont D. (1998) Histological study of retinal damages induced by multiple picosecond laser pulses, J. Laser Appl. 10, 229-231.

Courant D., Pérot J.-C.P., Garcia J., Paolacci-Riera S., Chapel C., Bourdon P., Dormont D. (1999a) Retinal damage thresholds induced by single and multiple picosecond laser pulses. In: International Laser Safety Conference, March 8-11, Orlando, Vol. 4, pp. 18-26. LIA, Orlando.

Courant D., Naudy-Vivès C., Perot J.-C., Garcia J., Dormont D. (1999b) Experimental determination of retinal damages thresholds induced by multiple picosecond laser pulses, J. Laser Appl. 11, 190-193.

Court L., Courant D., Guéneau G., Duchêne A., Laborde G. (1984) Effets du faisceau laser sur la fonction visuelle, Radioprotection 1 (Suppl.), 41-62.

FDA/CDRH (1985) Performance Standard for Laser Products, Center for Devices and Radiological health, Food and Drug Administration (DHHS), August 20, 50, pp. 33682-33702. Code of Federal Regulations (CFR). Washington.

Gertsman B.S., Thomson C.R., Jacques S.L., Rogers M.E. (1996) Laser induced bubble formation in the retina, Lasers Surg. Med. 18, 10-21.

Goldman A.L., Ham W.T., Mueller H.A. (1975) Mechanisms of retinal damage resulting from the exposure of rhesus monkeys to ultrashort laser pulses, Exp. Eye Res. 21, 457-469.

Goldman A.L., Ham W.T., Mueller H.A. (1977) Ocular damage thresholds and mechanisms for ultrashort pulses of both visible and infrared laser radiation in the rhesus monkey, Exp. Eye Res. 24, 457-469.

Griess G.A., Blankestein M.F., Williford G.G. (1980) Ocular damage from multiple pulses laser exposures, Health Phys. 39, 921-927. 


\section{RAYONNEMENT LASER}

Guéneau G.-V., Baille V., Courant D., Dubos M., Court L. (1986) Histopathology and ultrastructure of the retinal lesions induced on rabbits by single and low energy laser irradiations, Int. J. Radiat. Biol. 51, 925-926.

Ham W.T. Jr, Mueller H.A., Sliney D.H. (1976) Retinal sensitivity to damage from short wavelength light, Nature 260, 153-155.

Ham W.T., Mueller H.A., Ruffolo J.J., Clarke A.M. ( 1979 ) Sensitivity of the retina to radiation damage as a function of wavelength, Photochem. Photobiol. 29, 734-743.

Hammer D.X., Jansen E.D., Frenz M., Noojin G.D., Thomas R.J., Noack J., Vogel A., Rockwell B.A., Welch A.J. (1997) Shielding properties of laser induced breakdown in water for pulse durations from $5 \mathrm{~ns}$ to $125 \mathrm{fs}$, Appl. Opt. 36, 5630-5640.

Harwerth R.S., Sperling H.G. (1971) Prolonged color blindness induced by intense spectral light in rhesus monkeys, Science 174, 520-523.

IEC (1993) 825-I: Safety of laser products - Part 1: Equipment classification, requirements and user's guide. Bureau central de la Commission électrotechnique internationale, Genève.

ICNIRP, International Commission on Non Ionizing Radiation Protection (1996). Guidelines on limits for laser radiation of wavelengths between $180 \mathrm{~nm}$ and $1,000 \mu \mathrm{m}$, Health Phys. 71, 804-819.

ICNIRP, International Commission on Non Ionizing Radiation Protection (1997) Guidelines on limits of exposure for broad-band incoherent optical radiation $(0.38$ to $3 \mu \mathrm{m})$, Health Phys. 73, 539-554.

ICNIRP, International Commission on Non Ionizing Radiation Protection (2000) Revision of guidelines for laser radiation of wavelengths between $400 \mathrm{~nm}$ and $1.4 \mu \mathrm{m}$, Health Phys. (In press).

Jacques S.L., Glickman R.D., Schwartz J.A. (1996) Internal absorption coefficient and threshold for pulsed laser disruption of melanosomes isolated from retinal pigment epithelium. In: Laser-tissue interaction VII, January 29-February I, San Jose, SPIE, Vol. 2681 (Jacques S.L., Katzir A., Eds), pp. 468-477. Bellingham.

Lin C.P., Kelly M.W. (1998) Cavitation and accoustic emission around laser-heated microparticles, Appl. Phys. Lett. 72, 2800-2802.

Lund D.J. (1998) Bioeffects of near-infrared lasers, J. Laser Appl. 10, 140-143.

Marshall J. (1970) Thermal and mechanical mechanisms in laser damage to the retina, Invest. Ophthalmol. 9, 97-115.

Marshall J., Mellerio J. (1967) Disappearence of retino-epithelial scar tissue from ruby laser photocoagulations, Exp. Eye Res. 6, 4-9

Ness J.W., Zwick H., Stuck B.E., Lund D.J., Lund B.J., Molchany J.W., Sliney D.H. (2000) Retinal image motion during deliberate fixation: implication to laser safety for long duration viewing, Health Phys. 78, 131-142.

Noell W.K. (1980) Possible mechanisms of photoreceptor damage by light in mammalian eyes, Vision Res. 20, 1163-1171

Roach W.P., Toth C.A., Stein C.D., Noojin G.D., Stolarski D.J., Cain C.P. (1994) Minimum visible retinal lesions from pico- and femtosecond laser pulses. In: Laser-fissue interaction V, January 2427, San Jose, SPIE Vol. 2134A, (Jacques S.L., Katzir A., Eds), pp. 10-21. Bellingham.

Roach W.P., Johnson T.E., Rockwell B.A. (1999) Proposed maximum permissible exposure limits for ultrashort laser pulses, Health Phys. 76, 349-354.

Rockwell B.A., Hammer D.X., Hopkins R.A., Payne D.J., Toth C.A., Roach W.P., Druessel J.J., Kennedy P.K., Amnotte R.E., Eilert B., Phillips S., Noojin G.D., Stolarski D.J., Cain C.P. (1997) Ultrashort laser pulse bioeffects and safety pulses. In: International Laser Safety Conference, March 8- II, Orlando, Vol. 3, pp. 159-165. LIA, Orlando.

Sliney D.H., Wolbarsht M. (1980) Safety with lasers and other optical sources. Plenum Press, NewYork.

Sliney D.H. (1986) Physical factors in cataractogenesis-ambiant ultraviolet radiation and temperature, Invest. Ophthalmol. Vis. Sci. 27, 781-789. 


\section{COURANT et al.}

Sliney D.H. (1996) Laser effects on vision and ocular exposure limits, Appl. Occupational Environ. Hyg. 11, 313-319.

Sliney D.H. (1997) Evolving issues in laser safety, J. Laser Appl. 9, 295-300.

Sliney D.H. (1999) Status of the revision of the IEC standard. In: International Laser Safety Conference, March 8-11, Orlando, Vol. 4, pp. 166-179. LIA, Orlando.

Sperling H.G., Johnson C., Harwerth R.S. (1980) Differential spectral photic damage to primate cones, Vision Res. 20, 1117-1125

Strauss M., Amendt P., London R.A., Maitland D.S., Glinsky M.E., Lin C.P., Kelly M.W. (1997) Computational modeling of stress transient and bubble evolution in short pulse laser irradiated melanosome particles. In: Laser-tissue interaction VIII, Febuary 9-12, San Jose, SPIE Vol. 2975, (Jacques S.L., Katzir A., Eds), pp. 261-270. Bellingham.

Taboada J., Gibbons W.D. (1978) Retinal tissue damage induced by single ultrashort $1060 \mathrm{~nm}$ light pulses, Appl. Optics 17, 2871-2873.

Taylor H.R., West S.K., Rosenthal F.S., Munoz B., Newland H.S., Abbey H., Emmett E.A. (1988) Effect of ultraviolet radiation on cataract formation, New Engl. J. Med. 319, 1429-1433.

Thompson C.R., Gerstman B.S., Jacques S.L., Rogers M.E. (1996) Melanin granules model for laserinduced thermal damage in the retina, Bull. Math. Biol. 58, 513-553.

Toth C.A., Narayan D.G. Cain C.P., DiCarlo C.D., Rockwell B.A., Roach W.P. (1997) Histopathology of macular lesions from subnanosecond pulses of visible laser energy, Invest. Ophthalmol. Vis. Sci. 38, 2204-2213.

Toth C.A., Narayan D.G. Cain C.P., Noojin G.D., Winter K.P., Rockwell B.A., Roach W.P. (1996) Pathology of ultrashort laser pulse retinal damage, Invest. Ophthalmol. Vis. Sci. 37, 3170-3171.

Wolbarsht M.L. (1991) Cataract from infrared lasers: evidence for photochemical mechanisms, Lasers Light Ophthalmol. 4, 91-96.

Zuclich J.A., Lund D.J., Edsall P.R., Hollins R.C., Smith P.A., Stuck B.E., McLin L.N. (1999) Experimental study of the variation of laser-induced retinal damage threshold with retinal image size, Non linear Optics 21, 19-28.

Zwick H., Bloom K.A., Lund D.J., Beatrice E.S. (1983) Effects on minimal or punctate foveal lesions on visual acuity and contrast sensitivity in the Rhesus, Invest. Ophthalmol. Vis. Sci. 24, 296298.

Zwick H. (1989) Visual functional changes associated with low-level light effects, Health Phys. 56, 657-663. 